https://creativecommons.org/licenses/by/4.0/

\title{
IMPACTO EN LOS PROCESOS SOCIALES Y DE COMUNICACIONES EN UNA ENTIDAD ONCOLÓGICA COLOMBIANA ${ }^{1}$
}

\author{
Impact on social and communication processes \\ in a colombian cancer entity
}

\author{
GELLY GERALDINE ÁLVAREZ SUÁREZ², EVER ÁNGEL FUENTES ROJAS, MBA3, \\ ANDERSON STIWAR MURCIA GARCÍA
}

Recibido:06 de enero de 2020. Aceptado:15 de enero de 2020

DOI: $h$ ttp://dx.doi.org/10.21017/rimci.2020.v7.n13.a79

\begin{abstract}
Resumen
La entidad oncológica donde se realizó el estudio tiene como objetivo efectuar programas educativos de prevención y detección temprana en temas relacionados con el cáncer. En esta entidad, las áreas Social y de Comunicaciones son fundamentales para lograr dicho objetivo, ya que desarrolla cada función entorno a la sociedad para así potencializar estrategias que permitan la difusión a la comunidad y el acercamiento con familiares y pacientes, de tal manera que se promueve el intercambio de la información en temas de salud, prestándole mayor atención al cuidado, protección y la implementación de estilos de vida saludables. Dado lo anterior, se ha generado la necesidad de incorporar diferentes metodologías que permitan estandarizar los procesos que se llevan a cabo, midiendo el impacto y obteniendo como resultado oportunidades de mejora y eficacia.

Para este caso de estudio, se utilizaron herramientas de tipo ingenieril, obteniendo un diagnóstico inicial que parte desde la recolección de la información hasta la tabulación de la misma, con respecto a lo anterior, se toman decisiones estableciendo riesgos, factores, actividades y procedimientos que requieren mayor atención, no obstante, se realiza la respectiva documentación, incluyendo anexos e instructivos que permite entender a fondo el procedimiento y llevar un control del mismo mediante el análisis de tiempos no estructurados de cada actividad, por otro lado, se logra mitigar las diferentes amenazas e impactos negativos que puede tener frente a los cambios realizados, además, una mayor organización y control del proceso, generando un sistema de medición basado en indicadores que dará mayor soporte y control a lo desarrollado con su respectivo seguimiento, para finalizar se realizó un estudio de impacto que determina la viabilidad del proyecto, de acuerdo a lo anterior se pudo contemplar que la estandarización cuenta con varios factores positivos que ayudan al funcionamiento y cumplimiento de los objetivos misionales de la entidad oncológica y se verá reflejada en la atención a pacientes y apoyo a sus familiares, este proyecto respalda no solo la gestión documental sino la bonita labor que desarrolla.
\end{abstract}

Palabras clave. Estandarización; procesos; indicadores; riesgos; tiempos; salud.

\begin{abstract}
Oncological entities aim at training, prevention and education programs on cancer-related issues; the Social and Communications areas are fundamental to achieve this objective, since it exploits each function around society to thus potentiate strategies that can be dissemination, approach with family and patients, in such a way that the exchange of information on topics is promoted of health, paying greater attention to the care, protection and implementation of healthy lifestyles, given the above, the need to incorporate different methodologies that can standardize the processes that are carried out has been generated, resulting in opportunities for improvement and effectiveness.
\end{abstract}

1 Artículo producto del proyecto investigativo de la estandarización de procesos en el área Social y Comunicaciones de una entidad oncológica, ejecutado en el año 2018 y 2019 desde el programa de ingeniería de la Universidad Libre, sede Bogotá

2 Estudiante de ingeniería industrial. Universidad Libre, Colombia

3 Ingeniero industrial. Universidad Libre, Colombia. Docente de jornada completa del programa de ingeniería industrial. Universidad Libre, Colombia. Correo electrónico: ever.fuentes@unilibre.edu.co

4 Estudiante de ingeniería industrial. Universidad Libre, Colombia

Rev. Ingeniería, Matemáticas y Ciencias de la Información

Vol. 7 / Núm. 13 / enero - junio de 2020; pág. 91-103 
For this case study, use engineering tools obtaining an initial diagnosis, starting from the collection of information to the tabulation of the same, with respect to the above, making decisions establishing risks, factors, activities and procedures that diagnose more attention, however, the respective documentation is made, including annexes and instructions that allow a thorough understanding of the procedure and carry out a control of the same by analyzing unstructured times of each activity, on the other hand, it is possible to mitigate the different threats and negative impacts that may have to face the changes made, in addition, a larger organization and control of the process, generating a measurement system based on indicators that have greater support and control to what has been developed with their respective monitoring according to the stipulated to finalize an impact study was determined to determine the viability of the project or.

Keywords. Standardization; process; indicators; risk; times; health.

\section{INTRODUCCIÓN}

L es el foco central para brindar un mejor servicio, cuando se logra, proporciona una mayor eficiencia en los procesos que realizan, sin embargo, es necesario transformar el pensamiento, estar dispuesto a generar un cambio y potencializar el proceso hacia el cliente [1], es importante tener en cuenta que unos de los factores que afectan una organización es el desconocimiento de sus procesos, la falta de documentación y la escasez de indicadores, estas razones pueden ser un factor de amenaza de la entidad, debido a que pueden generar reprocesos, suplantación, incumplimiento de tareas y falta de soportes o seguimiento de los eventos o actividades que están relacionados directamente con el cumplimiento de la misión y visión.

Esta entidad oncológica tiene como objeto misional educar, prevenir y detectar el cáncer a tiempo, por esta razón se desarrollan: campañas, charlas educativas, talleres para el voluntariado en diferentes temas de promoción con médicos y expertos en el tema para brindar a la comunidad mejor información, desde diferentes puntos de vista. El cáncer es una enfermedad que puede tocar a cualquier individuo de la sociedad, por tal razón, es indispensable conocerla, detectarla a tiempo y tener claridad que por medio de chequeos médicos periódicos y hábitos de vida saludables se puede contrarrestar. En los casos en que la enfermedad se presenta, se debe recordar que el apoyo de las personas cercanas, el estado de ánimo es muy importantes en el proceso de sanación.

Dadas las circunstancia, la entidad oncológica decide fortalecer y mejorar sus objetivos, buscando beneficios para pacientes, familiares y la comunidad en general, con el desarrollo del proyecto de estandarización de procesos en las áreas, se pretende el mejoramiento en la prestación de ser- vicios, acompañamiento y soporte en el proceso de recuperación. El siguiente caso de estudio, se enfoca en la actualización y construcción documental de los procesos de estas dos áreas, encargadas directamente del contacto con la comunidad, con el fin de tener un mayor control, organización y eficacia en la ejecución de sus actividades dando cumplimiento a los objetivos planteados en cada procedimiento.

El contenido del artículo comprende las diferentes fases utilizadas para realizar un diagnóstico de la gestión documental, el desarrollo de un estudio de tiempos y movimientos de las operaciones de las áreas, la documentación de los procesos con la formulación de indicadores que permitiera un mayor control y validación de las actividades que integra los diferentes procedimientos, finalizando con un estudio de impacto en el cual se determinaría la viabilidad del proyecto.

El diagnóstico se realizó mediante entrevistas no estructuradas, lo cual permitió un acercamiento de las áreas, conociendo detalles, funcionamiento, objetivos, recursos y posibles mejoras en el estado documental, de igual manera, la caracterización estableció los diferentes procesos y planificación del sistema de gestión de calidad, mediante el alcance, los objetivos y los tipos de documentos requeridos por la organización, proveedores, detectando las entradas, salidas y relación con los clientes mediante la gestión documental planificada por los expertos [2]. De acuerdo con la información obtenida, se realizó un mapa de riesgos del proceso para poder identificar aquellos factores, causas y efectos más significativos inherentes y residuales a las actividades de las áreas, esta herramienta permitió validar y gestionar los riesgos que requieren mayor control mediante un plan de mejora [3]. Adicionalmente, la aplicación de la matriz DOFA y Vester, permitió distinguir factores internos y externos para la toma de decisiones. 
Para el estudio de tiempos y movimientos, se tomó la metodología de tiempos no estructurados con cronometro, se logró comprender las actividades mediante la estructuración de diagramas de operaciones en el cual se identificó actividades rutinarias y no rutinarias, a partir de lo anterior, se elaboró una tabulación de los tiempos tomados en el proceso y se obtuvo los tiempos estándar, dando alcance a los datos obtenidos se realizó un análisis en compañía de dos expertos que realizan actividades similares en diferentes áreas, se logra identificar el tiempo estructurado de una operación.

La elaboración de documentación se logró estructurar, tomando como referente la caracterización y un listado maestro de documentos de las áreas, de acuerdo con los resultados obtenidos, se establece los objetivos, alcance y pasos que componen el procedimiento, del mismo modo se realiza un diagrama de flujo en el cual se relacionan los diferentes instructivos, formatos, anexos y manuales que comprenden el mismo.

Partiendo del procedimiento y los objetivos se pudo establecer indicadores para realizar un seguimiento oportuno y según los resultados tomar decisiones o estrategias de mejora, finalizando el estudio se contempló un análisis de impacto mediante la estructuración de la matriz de Leopold lo que dio como resultado los factores positivos y negativos del proyecto.

\section{Marco teórico}

A continuación, se presentan los principales conceptos que se tuvieron en cuenta durante el desarrollo de la investigación:

a. Proceso: Es un conjunto de actividades relacionadas que interactúan y buscan la transformación de entradas en salidas para brindar un producto o servicio para el consumidor [4].

b. Características del proceso: Los elementos que identifican un proceso son:

1. Objetivo: Es el propósito, lo cual establece que se pretende lograr con él y se encuentra directamente relacionado con el producto final.
2. Responsable: Hace referencia a la persona que orienta, observa y mantiene el control, además se encarga de asignar los recursos necesarios para lograr el objetivo.

3. Alcance: Determina el inicio y fin.

4. Insumos: Los requerimiento del proceso en el que convierte las entradas en salidas mediante las actividades de transformación y utilización de los recursos.

5. Producto: Resultado final entregado a un cliente, debe satisfacer las necesidades identificadas en el servicio o producto.

6. Recursos: Todo aquello que permite el proceso de transformación de los insumos en el producto final.

7. Duración: Tiempo transcurrido desde la actividad identificada como inicio hasta la actividad final que es la entrega del producto final[5].

c. Estudio de tiempos: El estudio de tiempos es el procedimiento utilizado para medir el tiempo requerido por un trabajador calificado, quien trabajando a un nivel normal de desempeño realizando una tarea conforme a un método especificado [6].

1. Tiempo estándar: Según la Norma ANSI STANDARD Z94.0-1982, el valor de una unidad de tiempo para la realización de una tarea, como lo determina la aplicación apropiada de las técnicas de medición de trabajo efectuada por personal calificado. Por lo general se establece aplicando las tolerancias apropiadas al tiempo normal [7].

2. Tiempo normal: Es «el tiempo que requiere un operarlo calificado para realizar una tarea, a un ritmo normal, para completar un elemento, ciclo u operación usando un método prescrito» [7].

3. Tiempo básico: El tiempo básico se define como «tiempo mínimo irreducible que se calcula a partir de los tiempos elementales de una tarea de trabajo». Una tarea de trabajo es un conjunto de actividades necesarias para completar la 
ejecución de un proceso o producto. Cada tarea está compuesta de varios movimientos elementales[7].

d. Gestión: Actividades coordinadas para dirigir y controlar una organización [8].

e. Riesgo: Efecto de la incertidumbre, que es el estado de la deficiencia de información, relacionado con la compresión o conocimiento de un evento, consecuencia y probabilidades [8]. f. Mapa de riesgos: Herramienta que permite consolidad los riesgos identificados, analizados y evaluados en los procesos [9].

g. Mejora continua: Actividad recurrente para mejorar el desempeño [8].

h. Antecedentes: En la tabla I se mencionan distintas metodologías de proyectos similares, con el fin de tener una guía para el desarrollo de este. (Ver tabla I).

Tabla I. Antecedentes

\begin{tabular}{|c|c|c|}
\hline Nombre de los autores & Titulo & Descripción \\
\hline $\begin{array}{l}\text { Roberto Ravelo Mireles, Alberto } \\
\text { Martin Pérez Rodríguez, Maria } \\
\text { José Andrés Pardo, Pilar Campos } \\
\text { Esteban, Maria Eugenia Azpeitia } \\
\text { Serón, Ricardo Villarino Calvo }\end{array}$ & $\begin{array}{l}\text { Implementación local de la } \\
\text { estrategia de promoción de la } \\
\text { salud y prevención en el Sistema } \\
\text { Nacional de Salud. Informe } \\
\text { SESPAS } 2018\end{array}$ & $\begin{array}{l}\text { La estrategia de promoción y la prevención es una } \\
\text { prioridad en el Sistema Nacional de Salud, se con- } \\
\text { templó la necesidad de profundizar las acciones y } \\
\text { herramientas en la cuales se obtuvieron los resultados } \\
\text { esperados, permitiendo a las personas incrementar el } \\
\text { control sobre su salud y tomar hábitos que contribuyan } \\
\text { al mejoramiento, orientados a los estilo de vida que a } \\
\text { su vez indican la esperanza de esta. Se estableció un } \\
\text { marco común para la promoción que permita fortalecer } \\
\text { la salud en todas las políticas dando asi mayor } \\
\text { visibilidad a los recursos en salud comunitaria [10]. }\end{array}$ \\
\hline $\begin{array}{c}\text { Hans García Garcés, Lelys } \\
\text { Navarro Aguirre, Mayda López } \\
\text { Pérez, María de } \\
\text { Fátima Rodríguez Orizondo. }\end{array}$ & $\begin{array}{c}\text { Tecnologías de la Información y la } \\
\text { Comunicación en salud y } \\
\text { educación médica }\end{array}$ & $\begin{array}{l}\text { La tecnología ha creado nuevas forma de almacenar y } \\
\text { procesar información, esto conlleva a que los educa- } \\
\text { dores médicos deben estar muy preparados y atentos } \\
\text { para los cambios, debido al alto contenido de } \\
\text { información, conocimientos y técnicas, es necesario que } \\
\text { se tenga una habilidad de pensamiento crítico para } \\
\text { diferenciar la información veraz y científica. } \\
\text { Las TIC cada día crecen más y generan un gran } \\
\text { impacto en la salud y la educación médica, es } \\
\text { importante la adquisición de estas ya que conllevan a } \\
\text { una organización que sea más competitiva y la } \\
\text { satisfacción en la prestación de sus servicios a sus } \\
\text { clientes sea mayor debido a la actualización de } \\
\text { información hace que sea más efectiva y veraz [11]. }\end{array}$ \\
\hline $\begin{array}{l}\text { Ministerio de sanidad y política } \\
\text { social }\end{array}$ & $\begin{array}{l}\text { Implementación de guías de } \\
\text { práctica clínica en el sistema } \\
\text { Nacional de salud. Manual } \\
\text { metodológico }\end{array}$ & $\begin{array}{l}\text { El Ministerio de Sanidad y Política Social se refieren a } \\
\text { de una guía con el fin de establecer y conocer los } \\
\text { diferentes procesos, subprocesos que son utilizados en } \\
\text { la práctica clínica, las etapas utilizadas permiten la } \\
\text { selección de metodologías, levantamiento de infor- } \\
\text { mación, evaluación e implementación de indicadores } \\
\text { en la planificación del proceso y asi aumentar el } \\
\text { alcance que este tiene en cuanto al área Social } \\
\text { asumiendo todas las estrategias de implementación, } \\
\text { estableciendo una importante fuente de información } \\
\text { que alimente tanto la actualización e implementación } \\
\text { de futuros procesos de elaboración de guías [12]. }\end{array}$ \\
\hline
\end{tabular}

Fuente: Los autores, 2020

Rev. Ingeniería, Matemáticas y Ciencias de la Información Vol. 7 / Núm. 13 / enero - junio de 2020; pág. 91-103 


\section{Metodología}

Tras realizar una revisión literaria, enfocada en la estandarización, gestión documental, uso de herramientas y técnicas en las áreas Sociales y de Comunicaciones en entidades oncológicas o áreas afines, se evidenció la falta de investigaciones que hay acerca de este tema específico, pero aún más en el sector salud.

Para el desarrollo de este proyecto se aplicó un tipo de investigación mixto ya que se representa un conjunto de procesos sistemáticos, empíricos y críticos de investigación que implica la recolección, el análisis de datos cuantitativos y cualitativos, así como su integración y discusión conjunta [13]. Las características de mayor relevancia que representa una metodología de tipo mixto es que se puede contar con una perspectiva amplia y profunda lo cual implica recolección, análisis e integración de documentación que permita una variedad de información con el fin de establecer un criterio riguroso y sólido que sustenta el proyecto que se llevó a cabo en las áreas, logrando un mayor entendimiento del fenómeno bajo estudio.

Para la realización de este proyecto, se contó con metodologías y mecanismos que se muestra a continuación de acuerdo con la fase en desarrollo:

\section{A. Diagnóstico del área}

Inicialmente, se realizó una entrevista no estructurada, este tipo de encuestas cuenta con una serie de objetivos y está basada en el análisis, la ventaja de este tipo de herramientas es que se manejan como una conversación, además se pueden identificar diferentes factores y las preguntas se van generando a partir de la respuestas del entrevistado, es importante prestar mayor atención a los objetivos que tiene ya que se puede salir del contexto y puede desviar el mensaje central [14].

En este caso, se realizó una entrevista con el encargado de las áreas de manera individual y grupal, en la que se contempla los diferentes puntos de vista que tienen acerca de las áreas en cuanto a funciones, objetivos y procesos que se llevan a cabo, sin olvidar las funciones que tienen cada una y la experticia con la que manejan los temas según su profesión, por otro lado, se pudo identificar las diferentes relaciones que tiene los procedimientos con otras instituciones oncológicas, capítulos, pacientes y familiares ya que hacen parte fundamental de la prevención, educación y detección del cáncer. Con relación a lo anterior, se hizo un barrido de la documentación para establecer el estado de esta y se determinó que las áreas solo contaban con los soportes que se muestran en la Tabla II.

Tabla II. Gestión documental área Social y Comunicaciones

\begin{tabular}{|l|c|c|}
\hline \multirow{2}{*}{ Documentación } & \multicolumn{2}{|c|}{ Procedimiento } \\
\cline { 2 - 3 } & Campañas & Educación \\
\hline Procedimientos & 4 & 1 \\
\hline $\begin{array}{l}\text { Borrados de } \\
\text { procedimientos }\end{array}$ & 7 & 3 \\
\hline Formatos & 1 & 6 \\
\hline Anexos & 0 & 3 \\
\hline Guías & 0 & 2 \\
\hline \multicolumn{1}{|c|}{ TOTAL } & $\mathbf{1 2}$ & $\mathbf{1 5}$ \\
\hline
\end{tabular}

Fuente: Los autores, 2020

Los factores descritos fueron el foco central y se corroboró la información a través de la observación directa, luego se efectuó un diagrama de relaciones el cual es "una herramienta utilizada para desarrollar un contexto lógico en forma de ideas, opiniones, temas y aspectos a considerar" [15], este instrumento permitió identificar las relaciones causales existentes entre elementos, se logró organizar conceptos y actividades que fueron agrupadas por distintas categorías dando asi una estructuración de las áreas.

A medida que se logra entender y comprender un poco del área es importante hacer uso de metodologías que permitan identificar factores que se encuentren involucrados para alcanzar los objetivos misionales de la entidad, por esta razón se realiza una matriz DAFO para el análisis de diferentes escenarios que permite valorar las amenazas y oportunidades potenciales de un sector, proporciona estrategias corporativas o institucionales que son utilizadas en la ejecución de los planes de acción mediante métodos estratégicos para alcanzar los objetivos de las empresas mediante actividades y tareas, esta herramienta es aplicada para brindar soporte en la toma de decisiones [16]. 
Luego de elaborar la DAFO y obtener información, es necesario ampliar la visión de los datos obtenidos, por esta razón se considera realizar una matriz Vester ya que esta herramienta determina las causas y consecuencias en una situación problema, [17] allí se realiza una calificación de 0 a 3, donde 0 indica que no hay relación, 1 relación indirecta, 2 relación medianamente directa y 3 relación muy directa, luego de asignar la calificación, se deberán sumar filas y columna, de acuerdo a lo anterior, se puede conocer la influencia/ causa que tiene el problema sobre los factores descritos.

Para una mejor interpretación, se realiza un esquema axial [Fig. 1.] en la cual contemplan 4 ejes como los son pasivos, activos, críticos e inertes; como se muestra a continuación.

Se identifican los diferentes factores que tienen influencia y sensibilidad sobre los otros. Los cuadrantes se interpretan como activos, que cuenta con influencia intensa pero escasa sensibilidad, es decir se puede obtener una mejor orientación con respecto a los objetivos, pero se deben modificar los factores en forma directa o con ayuda de otros, por otro lado, los críticos cuentan con una alta influencia y sensibilidad, esto puede generar reacciones y efectos indeseados, se les debe prestar la mayor atención posible mediante monitoreos e intervenciones en el sistema [18], como bien se observa se encuentra un factor intermedio a la intersección entre pasivo y crítico, lo cual no representa mucha influencia, pero se debe solucionar para que no contribuya en un aspecto más por mejorar.

Al finalizar, el diagnóstico planteado, utilizar las herramientas y obtener la información acerca del proceso de las diferentes áreas, es necesario tomar medidas y empezar a implementar planes de mejora, documentar, actualizar y buscar solución a lo descrito.

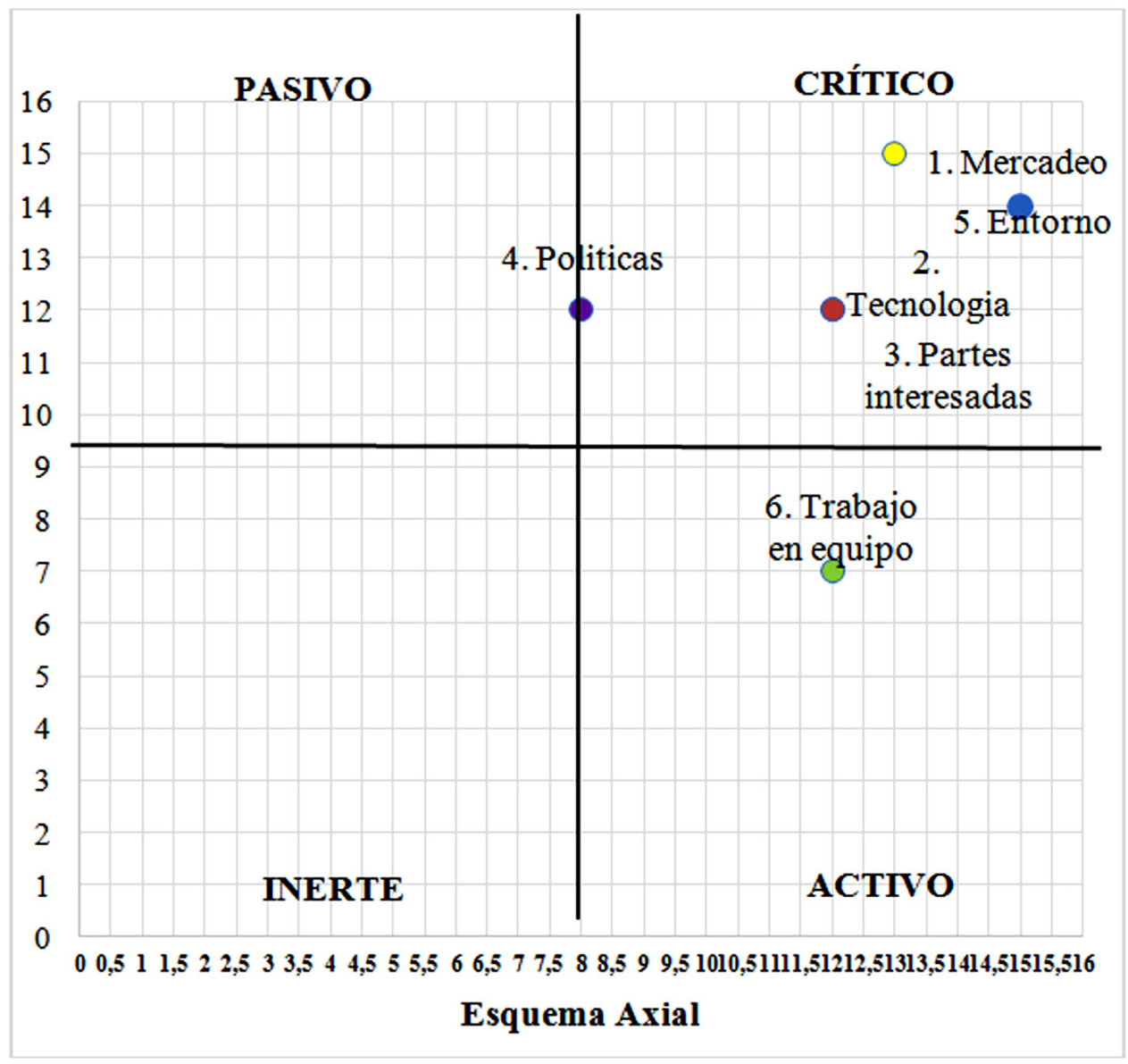

Fig.1. Matriz Vester. 2020. Fuente: Los autores 


\section{B. Documentación de procesos e implementación de indicadores}

Con base a los resultados se genera un listado maestro de documentos en el cual se establece los subprocesos, el tipo de instrumento (Procedimiento, instructivo, formato y manual), nombre, código de identificación de acuerdo a las políticas establecidas por parte de la entidad, versión, responsable del uso, persona que se encargará de revisar y aprobar la documentación; esto se realiza con el fin de entender y tener claridad de lo que se está ejecutando y lo que conlleva el cumplimiento de dichas actividades, luego de tener la información, se inicia la etapa de caracterización, es decir, una hoja de vida del proceso que consiste en identificar y clasificar el contenido en un modelo que permita organizar y comprender el mismo, es importante relacionar el tipo de proceso que se está manejando, es decir, si es estratégico, misional, apoyo y/o control, por otro lado, se debe contar con un objetivo, los responsables y el alcance, con el fin de establecer las entradas y salidas de proveedores y clientes, junto a la transformación de las actividades, verificando que se cumple con el ciclo PHVA, el cual comprende el planear, hacer, verificar y actuar [9].

Con base en la información obtenida, se procede a realizar un mapa de riesgos el cual comprende el proceso, subprocesos, procedimientos y los riesgos que conlleva cada uno, efectuando una clasificación del riesgo inherente con una probabilidad e impacto, lo cual establece la severidad del mismo que a su vez da paso a la inclusión de controles ya sea de tipo preventivo, correctivo y detectivo, esto conlleva a un plan de mejora que contrarreste o mitigue dicho factor que podría poner en riesgo la ejecución de actividades e inclusive el mismo proceso. Luego de contener esta tabulación se obtendrá una gráfica en la cual se podrá comprender de mejor manera esta información en [Fig. 2.]

Con respecto a la información previamente establecida, se realiza una clasificación el cual se divide en interno, externo, requisitos legales y los recursos con los que se cuenta, tales como el personal, la infraestructura y ambiente laboral que son de importancia para el conocimiento y orientación de los procesos de la organización. Es importante tener claridad que este trabajo es la base de la actualización y creación de documentos, sin embargo, en el transcurso del desarrollo de los mismo pueden sufrir algunos cambios o modificaciones que se registraran en la caracterización del proceso de las áreas, no obstante, no deberá afectar el foco central ni las actividades que se realizan allí.

Luego de contar con lo descrito anteriormente, se inicia el proceso de documentar ya sea el

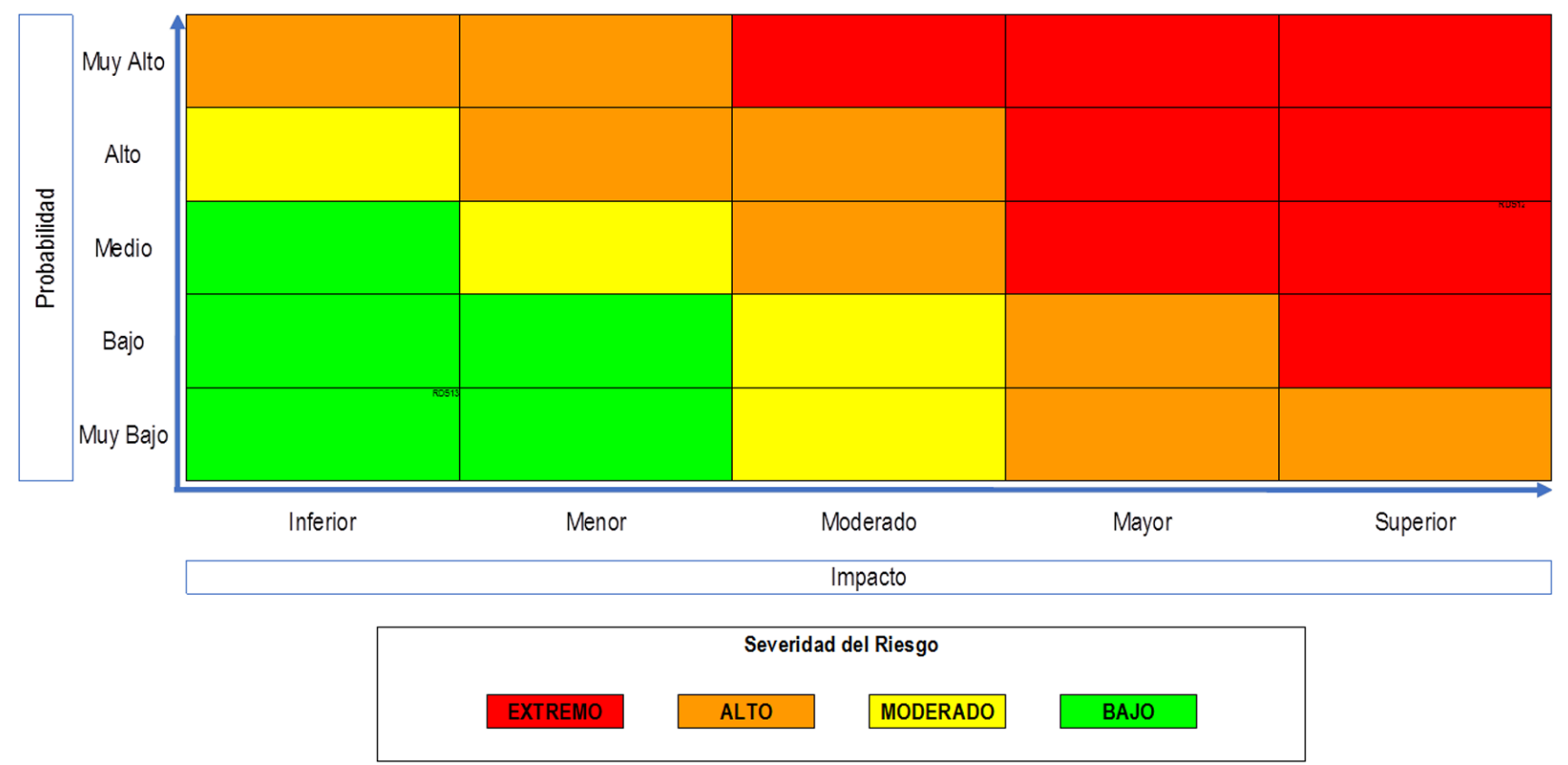

Fig.2. Matriz de riesgos. Fuente: Los autores con base a ISO 31000, 2020. 
procedimiento, instructivo, anexo, manual, según corresponda, es importante tener claro que todo documento cuenta con una estructura y ciertos parámetros definidos por la institución como se describe en la Tabla III.

De acuerdo a lo anterior y a medida que se van registrando los documentos con las indicaciones dadas, es importante contemplar la implementación de indicadores ya que es una herramienta cualitativa o cuantitativa que proporciona información de una situación, actividad o resultado que debe ser interpretado de una sola manera, cada indicador monitorea el objetivo al que se encuentra asociado, por otro lado, se deberá definir la dimensión de indicador ya sea eficacia, eficiencia, calidad y economía, cada uno contiene características diferentes, de acuerdo a lo que se mide y el cuándo se realiza este tipo de medición [19].

Por tal motivo, se contemplan los objetivos de las áreas para plantear de manera correcta los indicadores que estarán encaminado en el constante monitoreo de las actividades, estos siempre deberán contar con nombre, objetivo, alcance, variables, formulación y las fuentes de información que estarán ligadas directamente a la obtención de datos para contemplar las variables y así generar la medición correspondiente; cabe mencionar que al contar con cada uno de los indicadores se realizó un registro en la hoja de vida de ellos, el formato pertenece a la entidad dado que permite un mayor control y organización.

\section{Estudio de tiempos y movimientos}

El estudio de tiempos y movimientos, "es una herramienta que permite determinar los tiempos estándar de cada operación que compone el pro- ceso", Taylor y los esposos Gilberth fueron los precursores de esta técnica que tiene como objetivo planificar el trabajo mediante los tiempos estándar de una persona calificada que realiza el trabajo, proporcionando confiabilidad y alta calidad, reduciendo tiempos [20].

En el estudio de tiempos realizado en las áreas Social y de Comunicaciones, se inicia con el diagrama de operaciones que permite organizar las actividades del proceso como se muestra en la [Fig. 3].

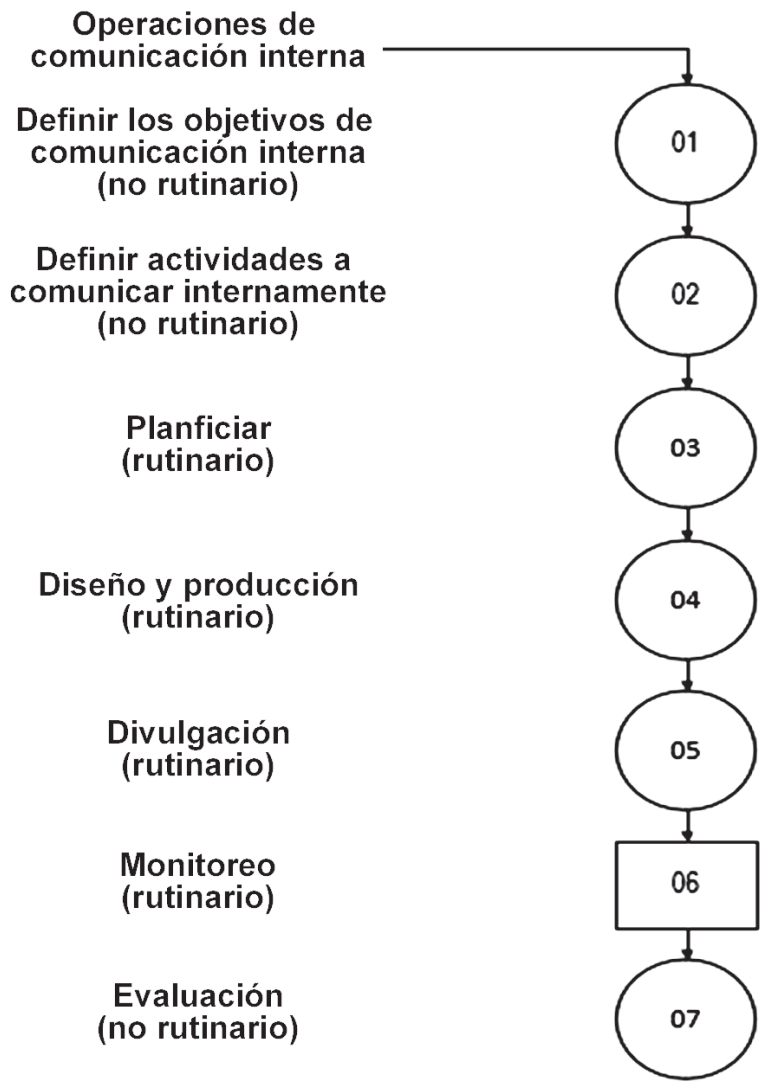

Fig. 3. Diagrama de operaciones. Fuente:

Tabla III. Contenido de documentación

\begin{tabular}{|c|c|c|c|c|}
\hline Sección & Manuales & Procedimientos & Instructivos & Formatos y anexos \\
\hline Objetivo & $\mathrm{Si}$ & $\mathrm{Si}$ & $\mathrm{Si}$ & No \\
\hline Alcance & $\mathrm{Si}$ & $\mathrm{Si}$ & $\mathrm{Si}$ & No \\
\hline Terminilogía y definiciones & Si Aplica & Si Aplica & Si Aplica & Si Aplica \\
\hline Procedimiento & Si Aplica & $\mathrm{Si}$ & Si Aplica & No \\
\hline Diagrama de flujo & No & Si Aplica & No & No \\
\hline Documentos relacionados & Si Aplica & Si Aplica & No & No \\
\hline Control de cambios & Si Aplica & Si Aplica & Si Aplica & No \\
\hline
\end{tabular}

Fuente: la entidad Oncológica, 2020 
Se toman cuatro muestras de tiempo para aplicar un método estadístico el cual requiere establecer el número de observaciones en las actividades rutinarias en condiciones similares mediante la ecuación (1).

$$
n=\left(\frac{\sqrt[40]{n^{\prime} \sum x^{2}-\sum(x)^{2}}}{\sum x}\right)^{2}(1)
$$

Siendo

$\mathrm{n}=$ Tamaño de la muestra que se desea calcular (número de observaciones)

$\mathrm{n}^{\prime}=$ Número de observaciones del estudio preliminar

$\Sigma=$ Suma de los valores

$\mathrm{x}=$ Valor de las observaciones

$40=$ Constante para un nivel de confianza de $94,45 \%$ luego de aplicar la fórmula

En la Tabla IV se observa un ejemplo de la tabulación de los tiempos y cálculo de las observaciones.

Luego de contar con dicha estructuración, se procede a realizar la toma de tiempos con el número de observaciones obtenidas para determinar un promedio de duración de ejecución de la actividad y posteriormente, se elabora un diagrama de dispersión con una línea de tendencia central con los datos tabulados.

Para las actividades no rutinarias se tuvo en cuenta tres tiempos que fueron manejados como "tiempos estructurados", el primero son los generados por la entidad, el segundo y tercero son establecidos por expertos del tema, con respecto a lo anterior, se da un porcentaje de confianza que será utilizado para obtener el tiempo estándar de la actividad (Tabla V).

\section{Estudio de impacto}

Para finalizar, se vio la necesidad de adaptar una herramienta que permitiera evaluar el impacto que tuvo el desarrollo del proyecto frente a las necesidades que se tenía y el cumplimiento de estas. Se consideró utilizar la matriz de Leopold, que permite evaluar acciones e interacciones de distintos factores, luego de determinar los posibles impactos se procede a realizar una evaluación en la que se contempla la magnitud en una escala de 1 a 10 donde 1 es la mínima alteración y 10 la máxima, se tienen en cuenta factores positivos y negativos que se encuentran relacionados con el signo $+\mathrm{o}-$, por otro lado, se habla de la importancia que se conforma mediante un peso relativo que se

Tabla IV. Cálculo de ciclos

\begin{tabular}{|c|c|c|c|c|c|c|c|c|}
\hline \multicolumn{6}{|c|}{ Comunicación interna } & $\Sigma(x)$ & $\Sigma(x)^{2}$ & n. \\
\hline 3 & Planificar & 58 & 54 & 57 & 56 & 225 & 12665 & 1 \\
\hline 4 & Diseño y producción & 41 & 45 & 42 & 40 & 168 & 7070 & 3 \\
\hline 5 & Divulgación & 26 & 30 & 28 & 29 & 113 & 3201 & 4 \\
\hline 6 & Monitoreo & 14 & 12 & 13 & 14 & 53 & 705 & 6 \\
\hline
\end{tabular}

Fuente: los autores, 2020

Tabla V. Tiempos estructurados

\begin{tabular}{|c|c|c|c|c|c|}
\hline \multicolumn{2}{|c|}{ Actividades } & 3 & 2 & $\mathbf{1}$ & Tiempo estándar \\
\hline 1 & $\begin{array}{c}\text { Definir los objetivos de } \\
\text { comunicación interna }\end{array}$ & 7200 & 10080 & 14400 & 9792 \\
\hline 2 & $\begin{array}{c}\text { Definir actividades a } \\
\text { comunicar internamente }\end{array}$ & 10080 & 10080 & 11520 & 10368 \\
\hline 7 & Evaluación & 135 & 120 & 180 & 138 \\
\hline
\end{tabular}

Fuente: Los autores, 2020 
considere dentro del proyecto o en algunos casos podrá presentar algunas interacciones [20].

\section{Resultados}

Tras la ejecución del proyecto, con la ayuda de los integrantes de las áreas y partes interesadas, se obtuvo diferentes resultados de acuerdo con las fases desarrolladas durante el transcurso de la estandarización.

Durante el diagnóstico se evidenció que en el área Social tenían la documentación desactualizada y en el área de Comunicaciones no se encontraba documentado los procesos que se realizan ni existen indicadores que permitiera el control y mejorar el enfoque. Se tiene en cuenta que las áreas siempre han sido manejadas por los mismos profesionales y su experticia ha permitido el funcionamiento y cumplimiento de las actividades que se planean a inicio de año, sin embargo, en el caso de no contar con estas personas podría haber afectaciones graves en la ejecución de las actividades que involucran directamente el cumplimiento del objetivo misional de la entidad, por esta razón se vio la necesidad de actualizar y documentar los procesos que se encontraron, presentando una propuesta innovadora que permita el control y organización en las áreas.

En la tabla VI se relacionan los documentos de cada área, se realiza la aclaración que el proceso de comunicaciones no contaba con ninguna actividad documentada ya que se estaba estructurando el área en el momento de realizar el proyecto.
Tabla VI. Documentación de las áreas

\begin{tabular}{|c|c|c|c|}
\hline \multicolumn{2}{|c|}{ Área social } & \multicolumn{2}{c|}{ Área comunicaciones } \\
\hline Procedimientos & 11 & Procedimientos & 4 \\
\hline Instructivos & $2-1$ video & Instructivos & $6-1$ video \\
\hline Formatos & 22 & Formatos & 11 \\
\hline Anexos & 2 & Anexos & 2 \\
\hline \multicolumn{2}{|c}{} & Manual & 1 \\
\cline { 2 - 4 }
\end{tabular}

Fuente: Los autores, 2020

Por otro lado, es importante destacar que las áreas se han preocupado por capacitar a personas y voluntariados, reconociendo a las personas que se encuentran vinculadas directa e indirectamente con las actividades, además se encuentra en mejora continua que, a pesar de no contar con todos los soportes, se actualizaron los procesos para mitigar los riesgos del entorno al cual han sido expuestos como se puede evidenciar en la Tabla VII y VIII.

Por otro lado, se realizó la formulación de indicadores que permitieran controlar, hacer un seguimiento del cumplimiento de objetivos y de actividades que requerian mayor atención, se lograron establecer y mostrar mediante el formato de hoja de vida suministrado directamente por la entidad oncológica (Fig. 4.) .

El anterior formato es una herramienta que permite la comprensión, manejo de información eficiente y totalmente comprensible, este cuenta con una hoja inicial donde se realiza la descripción y explicación de cada uno de los indicadores, además, la relación que tiene con los objetivos de cada proceso.

Tabla VII. Diagrama general del área Social

\begin{tabular}{|c|c|c|c|c|c|c|}
\hline \multicolumn{7}{|c|}{ Diagrama general - Severidad del riesgo } \\
\hline Área & & & a Socia & & & \\
\hline \multirow{2}{*}{ Subproceso } & \multirow{2}{*}{ Riesgos detectados } & \multicolumn{5}{|c|}{ Severidad del riesgo } \\
\hline & & Extremo & Alto & Moderado & Bajo & Total \\
\hline Capacitaciones extramurales & 2 & 0 & 2 & 0 & 0 & 2 \\
\hline Alianzas estratégicas & 5 & 3 & 1 & 1 & 0 & 5 \\
\hline Apoyo psicosocial & 4 & 1 & 1 & 1 & 1 & 4 \\
\hline Local & 1 & 0 & 1 & 0 & 0 & 1 \\
\hline Nacional & 3 & 1 & 2 & 0 & 0 & 3 \\
\hline Total & 15 & 5 & 7 & 2 & 1 & 15 \\
\hline
\end{tabular}

Fuente: Los autores, 2020 
IMPACTO EN LOS PROCESOS SOCIALES Y DE COMUNICACIONES EN UNA ENTIDAD ONCOLÓGICA COLOMBIANA GELLY GERALDINE ÁLVAREZ SUÁREZ, EVER ÁNGEL FUENTES ROJAS, ANDERSON STIWAR MURCIA GARCÍA

Tabla VIII. Diagrama general de Área de Comunicaciones

\begin{tabular}{|c|c|c|c|c|c|c|}
\hline \multicolumn{7}{|c|}{ Diagrama general - Severidad del riesgo } \\
\hline Área & & Con & nicaci & nes & & \\
\hline \multirow{2}{*}{ Subproceso } & \multirow{2}{*}{ Riesgos detectados } & \multicolumn{5}{|c|}{ Severidad del riesgo } \\
\hline & & Extremo & Alto & Moderado & Bajo & Total \\
\hline Comunicación interna & 3 & 1 & 0 & 2 & 0 & 3 \\
\hline Comunicación externa & 7 & 1 & 2 & 4 & 0 & 0 \\
\hline Total & 10 & 2 & 2 & 6 & 0 & 10 \\
\hline
\end{tabular}

Fuente: Los autores, 2020

\begin{tabular}{|c|c|c|c|c|}
\hline Macroproceso & \multicolumn{4}{|l|}{ Educación } \\
\hline Proceso: & \multicolumn{4}{|l|}{ Educación } \\
\hline Nombre: & \multicolumn{4}{|c|}{ Satisfacción en campañas educativas } \\
\hline Objetivo: & \multicolumn{4}{|c|}{$\begin{array}{l}\text { Medir la satisfacción de las campañas } \\
\text { realizadas estableciendo el fortalecimiento en } \\
\text { los temas presentados }\end{array}$} \\
\hline $\begin{array}{l}\text { Fórmula del } \\
\text { indicador }\end{array}$ & $\begin{array}{l}\text { Unidad } \\
\text { de } \\
\text { medida }\end{array}$ & $\begin{array}{c}\text { Nombre de las } \\
\text { variables }\end{array}$ & \multicolumn{2}{|c|}{$\begin{array}{l}\text { Fuente de } \\
\text { información }\end{array}$} \\
\hline $\begin{array}{c}\mathrm{N} .^{\circ} \text { de } \\
\text { personas } \\
\text { satisfechas con } \\
\text { las campañas } \\
\text { educativas / } \\
\mathrm{N} .^{\circ} \text { total de } \\
\text { personas } \\
\text { encuestadas }\end{array}$ & Unidades & $\begin{array}{c}\text { Numerador: } \\
\text { N. }{ }^{\circ} \text { de personas } \\
\text { satisfechas con } \\
\text { las campañas } \\
\text { educativas } \\
\text { Denominador: } \\
\text { N. } .^{\circ} \text { total de } \\
\text { personas } \\
\text { encuestadas }\end{array}$ & \multicolumn{2}{|c|}{$\begin{array}{l}\text { Formato de } \\
\text { evaluación de } \\
\text { campañas }\end{array}$} \\
\hline \multirow{4}{*}{$\begin{array}{l}\text { Periodicidad } \\
\text { de medición }\end{array}$} & Mensual & \multirow{4}{*}{$\begin{array}{c}\text { Periodicidad } \\
\text { de reporte }\end{array}$} & Mensual & \\
\hline & Trimestral & & Trimestral & \\
\hline & Semestral & & Semestral & \\
\hline & Anual & & Anual & $\mathbf{x}$ \\
\hline \multicolumn{5}{|c|}{ Partes interesadas } \\
\hline \multicolumn{5}{|l|}{$\begin{array}{l}\text { Entidades de } \\
\text { vigilancia y } \\
\text { control }\end{array}$} \\
\hline
\end{tabular}

\begin{tabular}{|l|l|l|l|l|l|}
\hline & Eficiencia & & & $\begin{array}{l}\text { Plan } \\
\text { estratégico } \\
\text { institucional }\end{array}$ & \\
$\begin{array}{l}\text { Tipo de } \\
\text { indicador }\end{array}$ & Eficacia & & $\begin{array}{l}\text { Nivel del } \\
\text { indicador }\end{array}$ & $\begin{array}{l}\text { Plan anual } \\
\text { de gestión }\end{array}$ & $\mathbf{X}$ \\
\cline { 2 - 3 } & Efectividad & $\mathbf{X}$ & & $\begin{array}{l}\text { Sistema } \\
\text { integral de } \\
\text { gestión }\end{array}$ & \\
\hline
\end{tabular}

\begin{tabular}{|l|c|c|c|c|c|c|}
\hline META & \multicolumn{6}{|c|}{ Umbral del 95\% } \\
\hline Rango & $\begin{array}{c}\text { Sobre- } \\
\text { saliente }\end{array}$ & $\begin{array}{c}95<=\text { meta } \\
<=\mathbf{1 0 0}\end{array}$ & $\begin{array}{c}\text { Acep- } \\
\text { table }\end{array}$ & $\begin{array}{c}\mathbf{8}<= \\
\text { meta } \\
<\mathbf{9 5}\end{array}$ & $\begin{array}{c}\text { Defici } \\
\text { ente }\end{array}$ & $\begin{array}{c}\text { Meta } \\
<80\end{array}$ \\
\hline
\end{tabular}

\begin{tabular}{|c|c|}
\hline $\begin{array}{c}\text { Responsabilidad de } \\
\text { recoger los datos }\end{array}$ & Área Social \\
\hline
\end{tabular}

Fig. 4. Hoja de vida de los indicadores. Fuente: La entidad oncológica, 2020
Para finalizar, fue necesario mostrar la viabilidad del proyecto realizado en las áreas, se generó un estudio de impacto y alcance, se tomaron diferentes factores que tuvieran relación con los procesos, en donde se estableció una calificación; los hallazgos positivos son el mayor número como se esperaba, estos fueron una oportunidad de mejora para las áreas e inclusive el conocimiento y claridad de los procesos ya que no se contaba con mucha información, no obstante, se realizaban de manera correcta solo que ahora se cuenta con soportes, información y claridad de los mismos, una medición y control de los factores importantes, por otro lado, los factores negativos solo abarca el $24 \%$ de las interacciones, donde se pude evidenciar que existe preocupación frente a los reprocesos pero este no se debe contemplar como una amenaza, al contrario, es una oportunidad de mejora que pretende dar cumplimiento y seguimiento a las actividades el proceso a ejecutar, son factores directamente relacionados a la adaptabilidad de los cambios que se presentan con la implementación de la estandarización.

\section{DisCusión DE RESUltados}

Se realizó una búsqueda de proyectos en el cual se evidenciara una estandarización de procesos en áreas similares, sin embargo, como se mencionó inicialmente se cuenta con pocos artículos con este tipo de proyectos. Se trabajó como referencia el documento del mapa de procesos del Ministerio de Salud, en donde se implementó la gestión de los procesos y mejora de los procedimientos, este caso de estudio parte de metodologías que permite identificar a los actores y productos a partir de una matriz, de acuerdo a lo anterior, se consideró recopilar

Rev. Ingeniería, Matemáticas y Ciencias de la Información

Vol. 7 / Núm. 13 / enero - junio de 2020; pág. 91-103 
información mediante entrevistas, informes técnicos con directores y asesores; luego se elaboró un mapa de procesos borrador en el que se establece los diferentes características y objetivos con relación a los roles, se realiza la descripción de cada proceso de nivel 0 , lo cual permitió describir los elementos que lo conforman, teniendo en cuenta el objetivo, alcance, responsable, requisitos, clasificación, proveedores, entradas, salidas e implementación de indicadores [21] [22]. Con relación a lo ejecutado en el proyecto se observó que el uso de metodologías son similares con respecto a la creación y documentación de procesos, teniendo en cuenta que se trata de tema normativo de calidad, no obstante, la estructura desarrollada en el proyecto de estandarización cuenta con mayor soporte frente al diagnóstico ya que se trabaja con menos sensibilidad a la información, el área social contaba con documentos desactualizados y en borrador que no habían sido aprobados, en cuanto a comunicaciones no contaba con los procedimientos establecidos ni con un estudio de tiempos, asimismo se demostró la viabilidad del proyecto mediante la matriz de Leopold que permite identificar las interacciones positivas que se obtuvieron en el caso de estudio.

\section{CONCLUSIONES Y RECOMENDACIONES}

De acuerdo con el trabajo realizado, el contenido de la información, el estudio que se llevó a cabo para culminar la estandarización de procesos en las áreas Social y de Comunicaciones permitió conocer más a fondo el proceso que se lleva a cabo en cada una de las áreas, se logró identificar riesgos y factores de amenaza que se logran mitigar a través del control y seguimiento.

El proceso ahora cuenta con soportes, información y claridad, una medición y control de los factores importantes mediante las diferentes herramientas y anexos incluidos en el proyecto, logrando comprender el alcance que tuvo el mismo; por otro lado, se recomienda seguir lo parametrizado con ayuda de las expertas de las actividades y los diferentes estudios que se hicieron para lograr la estandarización total de cada uno de los procedimientos y el control documental con el listado maestro, es importante, actualizar cada proceso que vaya cambiando con respecto al tiempo para no perder información ni el eje central de las áreas, se destaca la ardua labor que tienen y sobre todo la excelente misión que tienen para poder salvar vidas y mejorar los estilos de vida de pacientes y familiares.

\section{REFERENCIAS}

[1] A. Arias y F. d. C. d. 1. doucmentación, «Unidad didactica: 3, La gestión de los procesos,» [En línea]. Available: https://webs.ucm.es/centros/ cont/descargas/documento10142.pdf. [Último acceso: 25 Enero 2020].

[2] F. Becerra, A. Andrade y L. Diaz, Sistema de gestión de la calidad para el proceso de investigación, Ecuador: Universidad Otavalo, 2018.

[3] ISOtools, «¿En qué consiste una matriz de riesgos?,» 6 Agosto 2015. [En línea]. Available: https:/ /www.isotools.org/2015/08/06/en-que-consiste-una-matriz-de-riesgos/. [Último acceso: 25 Enero 2020].

[4] Instituto Colombiano de Normas Técnicas y Certificación (ICONTEC), «Norma técnica Colombiana NTC-ISO 9000,» Bogotá, 2005.

[5] L. F. Agudelo y J. Escobar, «Gestiión por procesos,» Instituto Colombiano de Normas Técnicas y Certificación, 2007, p. 306.

[6] C. Rincón y F. Villareal, «Contabilidad de costos I. Componentes del costo con aproximaciones a las NIC 02 y NIIF 08,» Ediciones de la U, Bogotá, 2014.

[7] Universidad de Costa Rica, «Tiempo estandar,» Escuela de administracion de negocios.

[8] ICONTEC, «Sistemas de gestión de la calidad-Fundamentos y vocabulario,» Bogotá, 2015.

[9] Alcaldia Mayor de Bogotá D.C., «Caracterización de procesos,» Bogotá, 2019.

[10] R. Ravelo, A. Martín, M. J. Pardo, P. Campos, E. Azpeitia y R. Villrino, «Implementación local de la Estrategia de Promoción de la Salud y Prevención en el Sistema Nacional de Salud. Informe SESPAS 2018,» Gaceta Sanitaria, vol. 32, nº 1, pp. 52-58, 2018.

[11] H. Garcia, L. Navarro, M. López y M. d. F. Rodriguez, «Tecnologías de la Información y la Comunicación en salud y educación médica,» Edumecentro, vol. 6, $\mathrm{n}^{\circ} 1,2014$.

[12] Ministerio de Salud y protección Social, «Manual de implementaciòn de guias de practica clinica basadas en evidencia, en instituciones prestadoras de servicios de salud,» Bogotá, 2014.

[13] R. Hernandez Sampieri, Metodologia de la investigación, Mexico: The McGraw-Hill, 2010.

Rev. Ingeniería, Matemáticas y Ciencias de la Información Vol. 7 / Núm. 13 / enero - junio de 2020; pág. 91-103 
[14] J. Casas, J. Repullo y J. Donado, «La encuesta como técnica de investigación. Elaboración de cuestionarios y tratamiento estadístico de los datos,» Atención primaria, vol. 31, $\mathrm{n}^{\circ}$ 8, pp. 527-538, 2003.

[15] J. Vilar, F. Gómez y M. Tejero, Las siete nuevas herramientas para la mejora de la calidad, FC Editorial, 1997.

[16] Z. Torres, Adminsitración estrategica, Mexico: Grupo Editorial Patria, 2014.

[17] J. Cuthbert, «Aplicación de la Matriz Vester,» [En línea]. Available: https:/ / e-aulas.urosario.edu.co/ pluginfile.php/676365/mod_folder/content/0/ Aplicaci \%C3\% B3n \% 20de \% 20la \% 20Matriz \% 20 Vester.doc?forcedownload=1. [Último acceso: 25 Enero 2020].
[18] Cooperación alemana; Giz; Reino de los Paises Bajos, Estrategias municipales de prevención de la violencia con participación de jóvenes, Porgrama regional GIZ-PREVENIR.

[19] Consejo Nacional de Evaluaciòn de la politica de Desarrollo Social , «Manual para el Diseño y la Construcción de Indicadores. Instrumentos principales para el monitoreo de programas sociales de México,» CONEVAL, Mexico, 2013.

[20] N. Tejada, V. Gisbert y A. Pérez, «Metodologia de estudio de tiempo y movimiento ; introducciòn al GSD,» 3C Empresa, pp. 39-49, 2017.

[21] S. Cotan, «Valoraciòn de impactos ambientaless,» INERCO, Sevilla, 2007.

[22] Oficina General de Planeamiento y Presupuesto; Grupo de Trabajo del Reglamento de Organización y Funciones del MINSA, «Mapa de Procesos del Ministerio de Salud,» Minsiterio de Salud, Perú, 2014. 
